\title{
A two-phase procedure for a multi-objective programming problem with fuzzy coefficients based on group decision- making for project selection
}

\author{
Miraç Eren ${ }^{1, \dagger}$ \\ ${ }^{1}$ Department of Economics, Faculty of Economics and Administrative Sciences, Ondokuz \\ Mayis University, 55060, Samsun, Turkey \\ E-mail:〈mirac.eren@omu.edu.tr>
}

\begin{abstract}
Regional Development Agencies (RDAs) play a major role in ensuring sustainability and reducing inter-regional and intra-regional development disparities in line with the principles and policies set in the National Development Plan and Programs. This is done by enhancing cooperation among the public and private sectors, as well as non-governmental organizations. To achieve these targets, RDAs use certain tools such as financial support programs, technical support programs, and the like. Accordingly, an effective evaluation mechanism is crucial in selecting projects that have more added value and higher multiplier effects. In this regard, determining the right parameters that assist in choosing the best projects should be clearly demonstrated. In this study, the selection of projects according to the evaluating criteria of support mechanisms considered by RDAs are discussed through the procedure provided by a practical solution methodology, which is an integration of fuzzy parametric programming (FPP) and fuzzy linear programming (FLP). Later, a two-phase procedure is introduced to solve multi-objective fuzzy linear programming problems.
\end{abstract}

Keywords: multiple objective programming, fuzzy parameters, project selection

Received: September 30,2016; accepted: March 30, 2017; available online: March 31, 2017

DOI: $10.17535 /$ crorr. 2017.0020

\section{Introduction}

Inter-regional and intra-regional development disparities are evident at different levels in the structures of all countries. Due to these disparities, all the countries face several social and economic problems. Many countries implement various solutions to overcome these challenges and succeed in establishing sustainable and balanced development. For this purpose, the first examples of solutions by Regional Development Agencies (RDAs) are from the 1930s. RDAs, generally conside-

${ }^{\dagger}$ Corresponding author 
red the best practical examples, especially in European Union countries, to an extent have made great contributions to the development of less developed regions. Hence, it is important to support SMEs (small and medium-sized enterprises), NGOs (non-governmental organizations), and public institutions, which play a considerable role in the development. However, due to the need for determining the right parameters and much insufficiency in the effective evaluation and monitoring mechanism, level of the needed analysis and support mechanism, have not been yet clearly identified. Therefore, choosing projects with added value, higher multiplier effects and less risk, in practice is the first step and has great importance in the development of regions and countries [23].

Traditional single criterion decision-making methods can no longer process the complexity of current systems in dealing with this problem [21]. Therefore, decision-making in selecting projects is viewed as multiple criteria decision-making (MCDM) problem with correlated criteria and alternatives. Multi-criteria decision analysis (MCDA) uses a flexible tool which helps bring a broad range of variables together, so that the decision maker may see the effects of the projects from a greater perspective and thus, make more accurate and precise (that is, more realistic) decisions. MCDM methods can be divided into two categories: multiobjective decision making (MODM) and multi-attribute decision making (MADM) [1].

The selection and evaluation of projects are based on subjective judgments. To overcome this situation, fuzzy sets theory is applied to MCDM problems [5, 16]. The natural language of perception or judgment is always subjective, uncertain or ambiguous [24]. Fuzzy logic is used for integrating imprecise data in decision making. Fuzzy sets can properly show uncertain parameters and can be managed through different operations on fuzzy numbers. Since uncertain parameters are treated as imprecise values instead of precise ones, the process will be stronger, and the results will be more credible [10].

Fuzzy MCDM (that is, MADM or MODM) methods related to project selection have frequently been discussed in the literature $[3,4,7,8,11]$. These studies focused on the selection of municipal, transportation and investment projects $[3,4,7$, 8]. There are also contributions dealing with multi-period project selection [11]. In the presence of different objectives and limitations, the use of multi-attribute decision-making (MADM) techniques provides a reliable methodology to generate rankings and select projects. However, in decision-making, it is difficult to state which technique is better. The reason for this, it is that the different methods can frequently produce different results, even when applied to the same problem using the same data. Even the normalization techniques employed in MADM methods can also give different ranking results. Therefore, it would be more appropriate if the selection or ranking decisions were considered as a constrained multi-objective optimization problem, not as a multi-attribute problem. Hence, one can then 
obtain more accurate and reliable results, without subjective judgments, or recourse to the normalization methods used to eliminate unit differences.

Conceptual techniques on the implementation of fuzzy optimization to multiobjective linear programming (MOLP) were firstly presented as fuzzy multiple objective decision-making problems by [27, 28]. After that, the solution procedures of fuzzy multiple objective decision-making problems were categorized into two main classes; first possibilistic and second probabilistic procedures [9]. According to [9], since membership functions are used to model the imprecise problem parameters in possibilistic procedures, possibility distributions which indicate the subjective and objective degree of the occurrence of an event are used in probabilistic procedures. Moreover, the multiple objective decision-making methods which consider all the parameters as fuzzy are known as possibilistic procedures (look at $[12,14,15,17,18,25])$ and the derivatives of these possibilistic procedures are also known as interactive procedures (look at [19, 20, 22]). The impreciseness has been modelled by these procedures, thanks to the possibility distributions which are generally presented by symmetric triangular/triangular and trapezoidal forms. However, the possibilistic procedures for optimization problems with fuzzy parameters have some disadvantages. Firstly, in expressing the fuzzy parameters mathematically, these procedures convert the linear model to a non-linear form. Thus, this non-linearity with an increase in the number of constraints and objectives has also increased the complexity of the models. As a result, solving becomes harder due to their computational complexity. Secondly, the possibilistic procedures put some restriction on the structures of membership functions as triangular, symmetric triangular and/or trapezoidal which are some of the wellknown probability distributions. Consequently, getting the implementable solutions with the flexibility of choosing the right expression of impreciseness of parameters, becomes more difficult.

In order to overcome such problems as mentioned above, in this study, the fuzzy parametric programming (FPP), being one of the fuzzy mathematical programming (FMP) methods, which had been proposed by [6] for single objective problems and afterwards improved by [2] for multiple objective problems, is adapted to construct a multiple objective decision problem according to the preferences reflecting the fuzziness derived from the decision-makers (DMs). Project selection procedures according to the evaluating criteria of support mechanisms considered by RDAs are discussed in developing the model. The criteria used by RDAs at present were determined in a Likert Scale and are composed of equally weighted five main-criteria. By considering the effects of subjective judgments on the criteria and alternatives, instead of using a single Likert scale number, fuzzy numbers are adopted to eliminate this ambiguity.

In the organization of the study, firstly, the multi-objective programming problem with fuzzy coefficients is discussed in the section "Material and Methods." Secondly, a resolution procedure for project selection by using a two-phase approach to 
the multi-objective programming problem with fuzzy coefficients is created in the section "Theory and Application of the Proposed Model." Finally, the findings are discussed in the section "Result and Discussions," and some suggestions are presented in the section "Conclusions."

\section{Material and methods}

\subsection{The multi-objective programming problem with fuzzy co- efficients}

The fuzzy parametric programming-FPP method can be adapted to set up multiple objective decision problems regarding different grades of precisions according to the preferences reflecting the fuzziness derived from the decision makers (DMs), it is possible to adapt. FPP, in which all coefficients in a mathematical model are considered as fuzzy, is different from other FMP methods $[2]$.

The proposed multi-objective FPP method provides the simultaneous optimal solutions for all objective functions or lexicographic optimal and/or additive solutions enabling different grades of precision according to the decision maker's preferences. All steps of the proposed procedure can be collected in two main stages: (a) the construction of a multi-objective FPP model for different precision levels and (b) solving multiple objective mathematical models by fuzzy linear programming (FLP).

\subsection{Solution procedure of the multi-objective FPP method}

Since the FLP method alone considers certain intervals, which are determined from the ideal solutions of the vector optimization model, the resulting solution is not dependent on DMs. However, this cannot be accepted as a disadvantage for the proposed procedure in this study, as the relative flexibility is reflected by using the mentioned FPP method. Therefore, the steps of the proposed multiobjective FPP method are:

Step 1: Setting up the multi-objective mathematical model The mathematical expression of the restricted multi-objective optimization problem is,

$$
\begin{array}{rc}
\operatorname{Max}(\operatorname{Min})\left\{z_{k}=f_{k}(x)=c_{k} x\right\} & k=1,2, \ldots, K \\
\text { subjects to: } \quad A x \leq . \geq .=b & x \geq 0
\end{array}
$$

where $f_{k}(x)$ are the kth objective functions, $z_{k}$ are objective function values, for all $k$ values, $c_{k}, x, b$ and $A$ respectively, represent the $1 x n$ dimensional vector of 
coefficients for all $\mathrm{k}$ values, the $m x 1$ dimensional vector of variables and $m x n$ dimensional coefficients matrix

Here, " $m$," " $n$ " and " $k$ " are the number of constraints, variables, and objective functions, respectively.

Step 2: Identifying fuzzy parameters

The suggested multi-objective FPP for restricted multiple objective equation systems where all parameters are fuzzy is a handy procedure. According to this procedure, the intervals for possible values of fuzzy parameters are specified by the user as $\left[c^{0}, c^{1}\right),\left[A^{0}, A^{1}\right)$ and $\left[b^{0}, b^{1}\right)$. Here, the lower bounds, which show the implementable solution, are called the "risk-free" zone and the upper bounds, which show the unimplementable solution, are called "impossible" zone. Starting from the "risk-free" zone to the "impossible" zone, the implementation ability decreases. In this context, the purpose of FPP is also to find the optimal compromise "in-between" solution as a function of grades of imprecision in parameters $[2,6]$.

The $\mu_{s}$ function, which represents the precision of an optimal solution, is expressed by the intersection of membership functions corresponding to imprecise parameters denoted by $\mu_{c}, \mu_{A}, \mu_{b}[6]$.

$$
\mu_{s}=\left(\mu_{c j} \cap \mu_{A i j} \cap \mu_{b i}\right), \quad i=1, \ldots, m, \quad j=1, \ldots, n .
$$

The given expression means that the inherited precision in the optimal solution equals the precision of the most "risky" of the parameters [6]. In equation (1), the best value for the objective function at a fixed level of $\mu_{s}$ is obtained when

$$
\mu_{s}=\left(\mu_{c j}=\mu_{A i j}=\mu_{b i}\right), \quad i=1, \ldots, m, \quad j=1, \ldots, n .
$$

This expression means that the best value of the objective function, at a fixed level of precision, can be found by using parameter values of the same level of precision, since the mathematical model tends to use the "risky" values of the parameters [6].

Step 3: Establishing membership functions

With the FPP method, fully trade-off membership functions are constructed in any possible form of the membership functions such as linear, piece-wise linear (triangular, trapezoidal), exponential or hyperbolic, and created by the imprecise parameters.

Step 4: Establishing restricted multi-objective decision problems based on the membership precision level

FPP models using constructed membership functions are created regarding precision level of fully trade-off memberships $\left(\mu=\left(\mu_{c k}\right.\right.$ or $\left.\left.\mu_{z k}\right)=\mu_{A}=\mu_{b}\right)$. The membership functions can have two possible alternative cases. By the way, each 
fuzzy parameter has identical membership functions and membership functions can be in different forms. In the first case, although the membership functions are non-linear, the FPP model can be easily solved by using traditional techniques. In the other case, alternative decision plans according to the grades of precision are presented after the model is solved for different values of the membership functions. For simplicity, under the assumption that all membership functions are in linear form and each model is a vector maximization problem, the FPP model for each $\mu$ level is

$$
\begin{gathered}
\max z_{k}=\left[c_{k}^{1}+\mu\left(c_{k}^{0}-c_{k}^{1}\right)\right] x, \quad k=1, \ldots, K \\
\text { subjects to: } \quad\left[A^{1}+\mu\left(A^{0}-A^{1}\right)\right] x \leq b^{1}+\mu\left(b^{0}-b^{1}\right) \\
x \geq 0
\end{gathered}
$$

Step 5: Identifying fuzzy intervals by using ideal solutions

Primarily, according to the precision level or for some preferred levels, the ideal solutions of the vector maximization problems are found. Later, to generate the payoff table, corresponding values of every objective at each solution derived are determined. Lastly, the best (u) and the worst (l) values for each objective corresponding to the set of solutions are obtained.

Step 6: Solutions generating by FLP

Multi-objective optimization has been applied to minimize or maximize functions with some constraints. This optimization technique enables an optimal solution with tradeoffs between two or more conflicting objectives. Therefore, it provided a compromising solution. In the literature, the approaches providing a compromising solution proposed for multi-objective optimization are the utility approach, goal programming, interactive approach, and fuzzy approach [26]. Among the investigations on the multi-objective linear programming problem, Zimmermann's fuzzy programming has been found to be an ideal procedure for obtaining the optimal compromising solution of a multi-objective problem [13]. The FLP method introduced by [28] formulates the fuzziness in both single and multiple objective problems. The FLP model, which optimizes the objectives simultaneously by finding the $[\mathrm{u}, \mathrm{l}]$, values of each objective regarding the vector maximization problem constructed for each precision levels, is

Subjects to:

$$
\operatorname{Max} \lambda
$$

$$
\begin{gathered}
\lambda \leq \frac{\left(z_{k}-l_{k}\right)}{\left(u_{k}-l_{k}\right)}, \quad k=1, \ldots, K, \\
{\left[A^{1}+\mu\left(A^{0}-A^{1}\right)\right] x \leq b^{1}+\mu\left(b^{0}-b^{1}\right)}
\end{gathered}
$$




$$
\begin{gathered}
0 \leq \lambda \leq 1, \\
x \geq 0,
\end{gathered}
$$

Where $\mu_{k}(x)=\frac{\left(z_{k}-l_{k}\right)}{\left(u_{k}-l_{k}\right)^{\prime}} \quad \mu=\mu_{z}=\mu_{A}=\mu_{b}=\mu_{c}$ and

$$
z_{k}=\left[c_{k}^{1}+\mu\left(c_{k}^{0}-c_{k}^{1}\right)\right] x, \quad \forall k .
$$

The solutions for each $\mu_{k}(x)$ value of equation (5) are combined using FLP. When the DM is not happy with the alternative decision plans containing each precision level, then the individual must repeat the procedure starting from step 2 or 3.

\section{The theory and application of the proposed model}

\subsection{Project selection using a two-phase approach for the multi-objective programming problem with fuzzy coefficients}

To illustrate how the proposed multi-objective FPP method works, this study was adopted from data in the article titled "Using Fuzzy Ahp and Fuzzy Topsis Methods for the Analysis of Development Agencies Project Evaluation Criteria" prepared by [23]. According to the study of [23] discussing the supports provided by Regional Development Agencies (RDAs) and the evaluating criteria of supports, the evaluation criteria for 20 project alternatives were grouped into five main aspects as C1- Financial and business capacity, C2- Relevancy, C3- Method, C4-Sustainability, C5- Budget and cost effectiveness, respectively. Also, three independent auditors evaluated it, and expert opinions were expressed using triangular fuzzy numbers. In this study, the multi-objective FPP method has been presented to suggest an alternative method to the RDAs for the selection of projects. Subsequently, the steps resulting from the proposed procedure are as follows:

\section{Setting up the multi-objective mathematical model}

The multi-objective mathematical model with fuzzy coefficients based on group decision-making for the 20 projects evaluated by different experts and considering each evaluation criteria as an aim is as follows:

\section{Indices}

$\mathrm{i}$ : the number of projects $(\mathrm{i}=1 \ldots, 20)$

$\mathrm{j}$ : the number of experts $(\mathrm{j}=1,2,3)$

\section{Parameters}

$\left[C 1_{i j}^{0}, C 1_{i j}^{1}\right)$ : the "risk-free" and "impossible" values of "Financial and business capacity" criteria evaluated by the jth expert for the ith project 
$\left[C 2_{i j}^{0}, C 2_{i j}^{1}\right)$ : the "risk-free" and "impossible" values of "Relevancy" criteria evaluated by the jth expert for the ith project

$\left[C 3_{i j}^{0}, C 3_{i j}^{1}\right)$ : the "risk-free" and "impossible" values of "Method" criteria evaluated by the jth expert for the ith project

$\left[C 4_{i j}^{0}, C 4_{i j}^{1}\right)$ : the "risk-free" and "impossible" values of "Sustainability" criteria evaluated by the jth expert for the ith project

$\left[C 5_{i j}^{0}, C 5_{i j}^{1}\right)$ : the "risk-free" and "impossible" values of "Budget and cost effectiveness" criteria evaluated by the jth expert for the ith project

\section{Decision variables}

$x_{i}$ : the weight value of the ith project (namely, this value is the degree of importance that indicates which project is better according to the existing parameters.)

\section{Objective functions and subjects}

* The total financial and business capacity of the projects

$$
\max z_{1}=\sum_{i=1}^{20} \sum_{j=1}^{3}\left[C 1_{i j}^{0}, C 1_{i j}^{1}\right) x_{i}
$$

* The total relevancy of the projects

$$
\max z_{2}=\sum_{i=1}^{20} \sum_{j=1}^{3}\left[C 2_{i j}^{0}, C 2_{i j}^{1}\right) x_{i}
$$

* The sum of method scores related to the projects

$$
\max z_{3}=\sum_{i=1}^{20} \sum_{j=1}^{3}\left[C 3_{i j}^{0}, C 3_{i j}^{1}\right) x_{i}
$$

* The total sustainability of the projects

$$
\max z_{4}=\sum_{i=1}^{20} \sum_{j=1}^{3}\left[C 4_{i j}^{0}, C 4_{i j}^{1}\right) x_{i}
$$

* The total Budget and cost effectiveness of the projects

$$
\max z_{5}=\sum_{i=1}^{20} \sum_{j=1}^{3}\left[C 5_{i j}^{0}, C 5_{i j}^{1}\right) x_{i}
$$

\section{Subject to:}

$$
\begin{aligned}
\sum_{\mathrm{i}=1}^{20} \mathrm{x}_{\mathrm{i}} & =1 \\
x_{i} & \geq 0, \quad \forall i=1, \ldots, 20
\end{aligned}
$$


Establishment of restricted multi-objective decision problems regarding membership precision level

For simplicity, under the assumption that membership functions regarding fuzzy parameters are in linear form and each aim is a vector maximization problem, the eleven different vector maximization models with a fixed level of precision carried out with various membership values are

$$
\left\{\begin{array}{l}
\text { for } \mu=0 \text { to } 1 \text { by } 0.1 \\
\max z_{1}=\sum_{i=1}^{20} \sum_{j=1}^{3}\left[C 1_{i j}^{1}+\mu\left(C 1_{i j}^{0}-C 1_{i j}^{1}\right)\right] x_{i} \\
\max z_{2}=\sum_{i=1}^{20} \sum_{j=1}^{3}\left[C 2_{i j}^{1}+\mu\left(C 2_{i j}^{0}-C 2_{i j}^{1}\right)\right] x_{i} \\
\max z_{3}=\sum_{i=1}^{20} \sum_{j=1}^{3}\left[C 3_{i j}^{1}+\mu\left(C 3_{i j}^{0}-C 3_{i j}^{1}\right)\right] x_{i} \\
\max z_{4}=\sum_{i=1}^{20} \sum_{j=1}^{3}\left[C 4_{i j}^{1}+\mu\left(C 4_{i j}^{0}-C 4_{i j}^{1}\right)\right] x_{i} \\
\max z_{5}=\sum_{i=1}^{20} \sum_{j=1}^{3}\left[C 5_{i j}^{1}+\mu\left(C 5_{i j}^{0}-C 5_{i j}^{1}\right)\right] x_{i} \\
\text { subject to: } \quad \sum_{i=1}^{20} x_{i}=1 \\
x_{i} \geq 0, \quad \forall i
\end{array}\right.
$$

Identifying fuzzy intervals by using ideal solutions

After the ideal solutions of each maximization aim regarding precision level were individually optimized under the constraint that the sum of the weight value of each project equals 1 , the corresponding values of every objective at each solution derived were determined, and thus, the payoff table was generated. 


\begin{tabular}{|c|c|c|c|c|c|c|}
\hline$\mu$ & & $\mathrm{z} 1$ & $\mathrm{z} 2$ & $\mathrm{z} 3$ & $\mathrm{z} 4$ & $\mathrm{z} 5$ \\
\hline \multicolumn{7}{|l|}{0.0} \\
\hline & 1st aim optimization & 29.4 & 28.2 & 24.5 & 25.7 & 25.5 \\
\hline & 2nd aim optimization & 29.4 & 28.2 & 24.5 & 25.7 & 25.5 \\
\hline & 3rd aim optimization & 27.7 & 25.4 & 26.6 & 26.0 & 27.0 \\
\hline & 4th aim optimization & 27.5 & 25.4 & 26.6 & 26.0 & 27.0 \\
\hline & 5th aim optimization & 23.8 & 24.4 & 23.5 & 23.3 & 27.5 \\
\hline & best value $(\mathrm{u})(\max )$ & 29.4 & 28.2 & 26.6 & 26.0 & 27.5 \\
\hline & worst value(l) (min) & 23.8 & 24.4 & 23.5 & 23.3 & 25.5 \\
\hline \multicolumn{7}{|l|}{0.1} \\
\hline & 1st aim optimization & 28.2 & 27.0 & 23.3 & 24.5 & 24.3 \\
\hline & 2nd aim optimization & 28.2 & 27.0 & 23.3 & 24.5 & 24.3 \\
\hline & 3rd aim optimization & 26.3 & 24.2 & 25.4 & 24.8 & 25.8 \\
\hline & 4th aim optimization & 26.3 & 24.2 & 25.4 & 24.8 & 25.8 \\
\hline & 5th aim optimization & 22.6 & 23.2 & 22.3 & 22.1 & 26.3 \\
\hline & best value (u) (max) & 28.2 & 27.0 & 25.4 & 24.8 & 26.3 \\
\hline & worst value(l) (min) & 22.6 & 23.2 & 22.3 & 22.1 & 24.3 \\
\hline \multicolumn{7}{|l|}{0.2} \\
\hline & 1st aim optimization & 27.0 & 25.8 & 22.1 & 23.3 & 23.1 \\
\hline & 2nd aim optimization & 27.0 & 25.8 & 22.1 & 23.3 & 23.1 \\
\hline & 3rd aim optimization & 25.1 & 23.0 & 24.2 & 23.6 & 24.6 \\
\hline & 4th aim optimization & 25.1 & 23.0 & 24.2 & 23.6 & 24.6 \\
\hline & 5th aim optimization & 21.4 & 22.0 & 21.1 & 20.9 & 25.1 \\
\hline & best value $(\mathrm{u})(\max )$ & 27.0 & 25.8 & 24.2 & 23.6 & 25.1 \\
\hline & worst value(l) $(\mathrm{min})$ & 21.4 & 22.0 & 21.1 & 20.9 & 23.1 \\
\hline \multicolumn{7}{|l|}{0.3} \\
\hline & 1st aim optimization & 25.8 & 24.6 & 20.9 & 22.1 & 21.9 \\
\hline & 2nd aim optimization & 25.8 & 24.6 & 20.9 & 22.1 & 21.9 \\
\hline & 3rd aim optimization & 23.9 & 21.8 & 23 & 22.4 & 23.4 \\
\hline & 4th aim optimization & 23.9 & 21.8 & 23 & 22.4 & 23.4 \\
\hline & 5th aim optimization & 20.2 & 20.8 & 19.9 & 19.7 & 23.9 \\
\hline & best value $(\mathrm{u})(\max )$ & 25.8 & 24.6 & 23.0 & 22.4 & 23.9 \\
\hline & worst value(l) (min) & 20.2 & 20.8 & 19.9 & 19.7 & 21.9 \\
\hline \multicolumn{7}{|l|}{0.4} \\
\hline & 1st aim optimization & 24.6 & 23.4 & 19.7 & 20.9 & 20.7 \\
\hline
\end{tabular}




\begin{tabular}{|c|c|c|c|c|c|c|}
\hline & 2nd aim optimization & 24.6 & 23.4 & 19.7 & 20.9 & 20.7 \\
\hline & 3rd aim optimization & 22.7 & 20.6 & 21.8 & 21.2 & 22.2 \\
\hline & 4th aim optimization & 22.7 & 20.6 & 21.8 & 21.2 & 22.2 \\
\hline & 5th aim optimization & 19.0 & 19.6 & 18.7 & 18.5 & 22.7 \\
\hline & best value (u) (max) & 24.6 & 23.4 & 21.8 & 21.2 & 22.7 \\
\hline & worst value(l) (min) & 19.0 & 19.6 & 18.7 & 18.5 & 20.7 \\
\hline \multicolumn{7}{|l|}{0.5} \\
\hline & 1st aim optimization & 23.4 & 22.2 & 18.5 & 19.7 & 19.5 \\
\hline & 2nd aim optimization & 23.4 & 22.2 & 18.5 & 19.7 & 19.5 \\
\hline & 3rd aim optimization & 21.5 & 19.4 & 20.6 & 20.0 & 21.0 \\
\hline & 4th aim optimization & 21.5 & 19.4 & 20.6 & 20.0 & 21.0 \\
\hline & 5th aim optimization & 17.8 & 18.4 & 17.5 & 17.3 & 21.5 \\
\hline & best value (u) (max) & 23.4 & 22.2 & 20.6 & 20.0 & 21.5 \\
\hline & worst value(l) (min) & 17.8 & 18.4 & 17.5 & 17.3 & 19.5 \\
\hline \multicolumn{7}{|l|}{0.6} \\
\hline & 1st aim optimization & 22.2 & 21.0 & 17.3 & 18.5 & 18.3 \\
\hline & 2nd aim optimization & 22.2 & 21.0 & 17.3 & 18.5 & 18.3 \\
\hline & 3rd aim optimization & 20.3 & 18.2 & 19.4 & 18.8 & 19.8 \\
\hline & 4th aim optimization & 20.3 & 18.2 & 19.4 & 18.8 & 19.8 \\
\hline & 5th aim optimization & 16.6 & 17.2 & 16.3 & 16.1 & 20.3 \\
\hline & best value (u) (max) & 22.2 & 21.0 & 19.4 & 18.8 & 20.3 \\
\hline & worst value(l) (min) & 16.6 & 17.2 & 16.3 & 16.1 & 18.3 \\
\hline \multicolumn{7}{|l|}{0.7} \\
\hline & 1st aim optimization & 21.0 & 19.8 & 16.1 & 17.3 & 17.1 \\
\hline & 2nd aim optimization & 21.0 & 19.8 & 16.1 & 17.3 & 17.1 \\
\hline & 3rd aim optimization & 19.1 & 17.0 & 18.2 & 17.6 & 18.6 \\
\hline & 4th aim optimization & 19.1 & 17.0 & 18.2 & 17.6 & 18.6 \\
\hline & 5th aim optimization & 15.4 & 16.0 & 15.1 & 14.9 & 19.1 \\
\hline & best value (u) (max) & 21.0 & 19.8 & 18.2 & 17.6 & 19.1 \\
\hline & worst value(l) $(\mathrm{min})$ & 15.4 & 16.0 & 15.1 & 14.9 & 17.1 \\
\hline \multicolumn{7}{|l|}{0.8} \\
\hline & 1st aim optimization & 19.8 & 18.6 & 14.9 & 16.1 & 15.9 \\
\hline & 2nd aim optimization & 19.8 & 18.6 & 14.9 & 16.1 & 15.9 \\
\hline & 3rd aim optimization & 17.9 & 15.8 & 17.0 & 16.4 & 17.4 \\
\hline & 4th aim optimization & 17.9 & 15.8 & 17.0 & 16.4 & 17.4 \\
\hline
\end{tabular}




\begin{tabular}{|l|l|c|c|c|c|c|}
\hline & 5th aim optimization & 14.2 & 14.8 & 13.9 & 13.7 & 17.9 \\
\hline & best value (u) (max) & 19.8 & 18.6 & 17.0 & 16.4 & 17.9 \\
\hline & worst value(l) (min) & 14.2 & 14.8 & 13.9 & 13.7 & 15.9 \\
\hline 0.9 & & & & & & \\
\hline & 1st aim optimization & 18.6 & 17.4 & 1.7 & 14.9 & 14.7 \\
\hline & 2nd aim optimization & 18.6 & 17.4 & 1.7 & 14.9 & 14.7 \\
\hline & 3rd aim optimization & 16.7 & 14.6 & 15.8 & 15.2 & 16.2 \\
\hline & 4th aim optimization & 16.7 & 14.6 & 15.8 & 15.2 & 16.2 \\
\hline & 5th aim optimization & 13.0 & 13.6 & 12.7 & 12.5 & 16.7 \\
\hline & best value (u) (max) & 18.6 & 17.4 & 15.8 & 15.2 & 16.7 \\
\hline & worst value(l) (min) & 13.0 & 13.6 & 1.7 & 12.5 & 14.7 \\
\hline 1.0 & & & & & & \\
\hline & 1st aim optimization & 17.4 & 16.2 & 12.5 & 13.7 & 13.5 \\
\hline & 2nd aim optimization & 17.4 & 16.2 & 12.5 & 13.7 & 13.5 \\
\hline & 3rd aim optimization & 15.5 & 13.4 & 14.6 & 14.0 & 15.0 \\
\hline & 4th aim optimization & 15.5 & 13.4 & 14.6 & 14.0 & 15.0 \\
\hline & 5th aim optimization & 11.8 & 12.4 & 11.5 & 11.3 & 15.5 \\
\hline & best value (u) (max) & 17.4 & 16.2 & 14.6 & 14.0 & 15.5 \\
\hline & worst value(l) (min) & 11.8 & 12.4 & 11.5 & 11.3 & 13.5 \\
\hline
\end{tabular}

Table 1: Payoff table enabling an optimal solution with tradeoffs between two or more conflicting objectives regarding the values of the $\mu$ precision level

\subsection{Solutions generating by FLP}

The best (u) and the worst (l) values for each objective corresponding to the set of solutions using the values from the payoff table were obtained. Then, the FLP model, i.e. the latest version of the problem, is defined as 


$$
\begin{gathered}
\text { for } k=1 \text { to } 11 \\
\max \lambda \\
\text { subject to: } \\
\lambda \leq\left(z_{1}-l_{k}\right) /\left(u_{k}-l_{k}\right) \\
\lambda \leq\left(z_{2}-l_{k}\right) /\left(u_{k}-l_{k}\right) \\
\lambda \leq\left(z_{3}-l_{k}\right) /\left(u_{k}-l_{k}\right) \\
\lambda \leq\left(z_{4}-l_{k}\right) /\left(u_{k}-l_{k}\right) \\
\lambda \leq\left(z_{5}-l_{k}\right) /\left(u_{k}-l_{k}\right) \\
\\
\quad \sum_{i=1}^{20} x_{i}=1 \\
\text { end } \\
x_{i} \geq 0, \quad \forall i
\end{gathered}
$$

Where $\mathrm{k}$ is the number of solutions corresponding to each $\mu$ precision level value in the payoff table and $\lambda$ is a suitable variable that satisfies the definition of the operational adequacy measure, for the case that the objectives are hazy or cloudy. That is, the numerical value of $\lambda$ means that all objectives are optimized together, and one of them is satisfied with $\lambda$ the level and the others are satisfied at least at $\lambda$ level.

The results of FLP Models, which are created and solved for each precision level, provide alternative decision plans to the DMs/analysts.

\section{Results and discussions}

To determine the projects given the supports provided by Regional Development Agencies (RDAs) according to the specific evaluation criteria, the result of the FLP model is defined in Table 2.

\begin{tabular}{|c|c|c|c|c|c|c|c|c|c|c|c|c|c|c|c|}
\hline$\mu$ & $\lambda$ & $\mathrm{x}_{1}$ & $\mathrm{x}_{2}$ & $\mathrm{x}_{3}$ & $\mathrm{x}_{4}$ & $\mathrm{x}_{5}$ & $\mathrm{x}_{6}$ & $\mathrm{x}_{7}$ & $\mathrm{x}_{8}$ & $\mathrm{x}_{9}$ & $\mathrm{x}_{10}$ & $\mathrm{x}_{11}$ & $\mathrm{x}_{12}$ & $\mathrm{x}_{13}$ & $\mathrm{x}_{14}$ \\
\hline 0 & 0.50 & 0 & 0 & 0 & 0 & 0 & 0 & 0 & 0 & 0 & 0 & 0 & 0 & 0 & 0 \\
\hline 0.1 & 0.05 & 0 & 0 & 0 & 0 & 0 & 0 & 0 & 0 & 0 & 0 & 0 & 0 & 0 & 0 \\
\hline 0.2 & 0 & 0 & 0 & 0 & 0 & 0 & 0 & 0 & 0 & 0 & 0 & 0 & 0 & 0 & 0 \\
\hline 0.3 & 0 & 0 & 0 & 0 & 0 & 0 & 0 & 0 & 0 & 0 & 0 & 0 & 0 & 0 & 0 \\
\hline 0.4 & 0 & 0 & 0 & 0 & 0 & 0 & 0 & 0 & 0 & 0 & 0 & 0 & 0 & 0 & 0 \\
\hline 0.5 & 0 & 0 & 0 & 0 & 0 & 0 & 0 & 0 & 0 & 0 & 0 & 0 & 0 & 0 & 0 \\
\hline
\end{tabular}




\begin{tabular}{|c|c|c|c|c|c|c|c|c|c|c|c|c|c|c|c|}
\hline 0.6 & 0 & 0 & 0 & 0 & 0 & 0 & 0 & 0 & 0 & 0 & 0 & 0 & 0 & 0 & 0 \\
\hline 0.7 & 0 & 0 & 0 & 0 & 0 & 0 & 0 & 0 & 0 & 0 & 0 & 0 & 0 & 0 & 0 \\
\hline 0.8 & 0 & 0 & 0 & 0 & 0 & 0 & 0 & 0 & 0 & 0 & 0 & 0 & 0 & 0 & 0 \\
\hline 0.9 & 0 & 0 & 0 & 0 & 0 & 0 & 0 & 0 & 0 & 0 & 0 & 0 & 0 & 0 & 0 \\
\hline 1.0 & 0 & 0 & 0 & 0 & 0 & 0 & 0 & 0 & 0 & 0 & 0 & 0 & 0 & 0 & 0 \\
\hline
\end{tabular}

\begin{tabular}{|c|c|c|c|c|c|c|c|}
\hline$\mu$ & $\lambda$ & $\mathrm{x}_{15}$ & $\mathrm{x}_{16}$ & $\mathrm{x}_{17}$ & $\mathrm{x}_{18}$ & $\mathrm{x}_{19}$ & $\mathrm{x}_{20}$ \\
\hline 0 & 0.50 & 0.67 & 0 & 0 & 0 & 0 & 0.33 \\
\hline 0.1 & 0.05 & 0.86 & 0 & 0 & 0 & 0 & 0.14 \\
\hline 0.2 & 0 & 1.00 & 0 & 0 & 0 & 0 & 0 \\
\hline 0.3 & 0 & 1.00 & 0 & 0 & 0 & 0 & 0 \\
\hline 0.4 & 0 & 1.00 & 0 & 0 & 0 & 0 & 0 \\
\hline 0.5 & 0 & 1.00 & 0 & 0 & 0 & 0 & 0 \\
\hline 0.6 & 0 & 1.00 & 0 & 0 & 0 & 0 & 0 \\
\hline 0.7 & 0 & 1.00 & 0 & 0 & 0 & 0 & 0 \\
\hline 0.8 & 0 & 1.00 & 0 & 0 & 0 & 0 & 0 \\
\hline 0.9 & 0 & 1.00 & 0 & 0 & 0 & 0 & 0 \\
\hline 1.0 & 0 & 1.00 & 0 & 0 & 0 & 0 & 0 \\
\hline
\end{tabular}

Table 2: FLP results created and solved for $\mu=0,0.1, \ldots, 1$

According to the aggregated satisfaction level of the FLP models for each precision level $(\mu=0,0.1, \ldots, 1)$, the best satisfaction level of the aims is equal to 0.5 , and this has been provided with the value of $\mu=0$. As a result, the generated mathematical model has assigned $67 \%$ and $33 \%$ weight values for the 15th and 20 th project, respectively. Also, there are alternative decision plans for other $\mu$ values.

Twenty projects according to the five evaluating criteria considered by RDAs were evaluated based on the opinions of three independent auditors. However, in this research, the institution's resources were not taken into account. If institution's resources were considered, this would have had to be added as a constraint to the mathematical model created. Perhaps then, the institution's resources would have allowed it to support another project.

\section{Conclusions}

In this research, implementation of a new mathematical procedure in multi-objective decision problems with fuzzy coefficients for project selection is introduced. The paper presents an overview of fuzzy linear programming and fuzzy parametric 
programming, which are two well-known techniques. A new procedure has been established and explained along with all the details and illustrated using a simple numerical step-by-step example. All the data used in this study has been collected from the DM's experience, information and expecta-tions through interaction. The solutions at each precision level and obtained from the model are optimal and efficient. This procedure can be used in a wide range of real-life problems. We have two reasons to support this idea. First, handling the imprecision of parameters is very easy in the multiobjective mathematical programming (MOMP) context, and secondly, the solution characteristics of the procedure have no effect on the complexity of mathematical models. Therefore, we believe that the procedure can be applied to many real-life problems.

\section{References}

[1] Abu-Taha, R. (2011). Multi-criteria applications in renewable energy analysis: a literature review Technology Management in the Energy Smart World (PICMET). 2011 Proceedings of PICMET '11: , 1-8.

[2] Arıkan, F.and Güngör, Z. (2007). A two-phase approach for multi-objective programming problems with fuzzy coefficients. Information Sciences, 177 5191-5202.

[3] Avineri, E., Prashker, J.andCeder, A. (2000). Transportation projects selection process using fuzzy sets theory. Fuzzy Sets and Systems, 116 35-47.

[4] Baysal, M.E., Kaya, İ., Kahraman, C., Sarucan, A.and Engin, O. (2015). A two phased fuzzy methodology for selection among municipal projects. Technological and Economic Development of Economy, 21 405-422.

[5] Büyüközkan, G., Feyzioğlu, O.and Nebol, E. (2008). Selection of the strategic alliance partner in logistics value chain. International Journal of Production Economics, 113 148-158.

[6] Carlsson, C.and Korhonen, P. (1986). A parametric approach to fuzzy linear programming. Fuzzy Sets and Systems, 20 17-30.

[7] Ferrari, P. (2003). A method for choosing from among alternative transportation projects. European Journal of Operational Research, 150 194-203.

[8] Huang, X. (2007). Optimal project selection with random fuzzy parameters. International Journal of Production Economics, 106 513-522.

[9] Jou Lai, Y.and Hwang, C. (1994). Fuzzy Multiple Objective Decision Making, Methods and Applications, in. Springer-verlag Berlin Heidelberg.

[10] Kahraman, C. (2006). Fuzzy Applications in Industrial Engineering, Springer.

[11] Khalili-Damghani, K.and Sadi-Nezhad, S. (2013). A decision support system for fuzzy multi-objective multi-period sustainable project selection. Computers and Industrial Engineering, 64 1045-1060. 
[12] Lai, Y.-J.and Hwang, C.-L. (1992). A new approach to some possibilistic linear programming problems. Fuzzy Sets and Systems, 49 121-133.

[13] Li, L.and Lai, K.K. (2000). A fuzzy approach to the multiobjective transportation problem. Computers and Operations Research, 27 43-57.

[14] Li, R.and Lee, E. (1990). Multi-criteria de novo programming with fuzzy parameters. Computers and Mathematics with Applications, 19 13-20.

[15] Negi, D.S. 1996. Fuzzy Analysis and Optimization, UMI.

[16] Paksoy, T., Pehlivan, N.Y.and Kahraman, C. (2012). Organizational strategy development in distribution channel management using fuzzy AHP and hierarchical fuzzy TOPSIS. Expert Systems with Applications, 39 2822-2841.

[17] Parra, M.A., Terol, A.B., Gladish, B.P.and Uria, M.R. (2005). Solving a multiobjective possibilistic problem through compromise programming. European Journal of Operational Research, 164 748-759.

[18] Parra, M.A., Terol, A.B.and Urıa, M.R. (1999). Solving the multiobjective possibilistic linear programming problem. European Journal of Operational Research, 117 175-182.

[19] Rommelfanger, H. (1989). Interactive decision making in fuzzy linear optimization problems. European Journal of Operational Research, 41 210-217.

[20] Sakawa, M.and Yano, H. (1989). An interactive fuzzy satisficing method for multiobjective nonlinear programming problems with fuzzy parameters. Fuzzy Sets and Systems, 30 221-238.

[21] San Cristóbal, J. (2011). Multi-criteria decision-making in the selection of a renewable energy project in Spain: the Vikor method. Renewable Energy, 36 498-502.

[22] Słowiński, R. (1986). A multicriteria fuzzy linear programming method for water supply system development planning. Fuzzy Sets and Systems, 19 217237.

[23] Söyler, H.and Pirim, L. (2014). Using fuzzy ahp and fuzzy topsis methods for the analysis of development agencies project evaluation criteria. NWSA: Social Sciences, 9 105-117.

[24] Wang, T.-C.and Chang, T.-H. (2007). Application of TOPSIS in evaluating initial training aircraft under a fuzzy environment. Expert Systems with Applications, 33 870-880.

[25] Wierzchon, S. (1987). Linear programming with fuzzy sets: a general approach. Mathematical Modelling, 9 447-459.

[26] Yaralıŏ̆lu, K.and Umarusman, N. (2010). Çok Amaçlı Doğrusal Programlamadan Sistem Tasarımına: De Novo. Dokuz Eylül Üniversitesi Sosyal Bilimler Enstitüsü Dergisi, 12.

[27] Zimmermann, H.-J. (1975). Description and optimization of fuzzy systems. International Journal of General System, 2 209-215.

[28] Zimmermann, H.-J. (1978). Fuzzy programming and linear programming with several objective functions. Fuzzy Sets and Systems, 1 45-55. 